\title{
Photocontrolled reversible self-assembly of dodecamer nitrilase
}

\author{
Qiao Yu' ${ }^{1}$, Yong Wang ${ }^{2}$, Shengyun Zhao ${ }^{3}$ and Yuhong Ren ${ }^{*}$
}

\begin{abstract}
Background: Naturally photoswitchable proteins act as a powerful tool for the spatial and temporal control of biological processes by inducing the formation of a photodimerizer. In this study, a method for the precise and reversible inducible self-assembly of dodecamer nitrilase in vivo (in Escherichia coli) and in vitro (in a cell-free solution) was developed by means of the photoswitch-improved light-inducible dimer (iLID) system which could induce proteinprotein dimerization.

Results: Nitrilase was fused with the photoswitch protein AsLOV2-SsrA to achieve the photocontrolled self-assembly of dodecamer nitrilase. The fusion protein self-assembled into a supramolecular assembly when illuminated at $470 \mathrm{~nm}$. Scanning electron microscopy showed that the assembly formed a circular sheet structure. Self-assembly was also induced by light in E. coli. Dynamic light scattering and turbidity assay experiments showed that the assemblies formed within a few seconds under 470-nm light and completely disassembled within 5 min in the dark. Assembly and disassembly could be maintained for at least five cycles. Both in vitro and in vivo, the assemblies retained $90 \%$ of the initial activity of nitrilase and could be reused at least four times in vitro with $90 \%$ activity.

Conclusions: An efficient method was developed for the photocontrolled assembly and disassembly of dodecamer nitrilase and for scaffold-free reversible self-assembly of multiple oligomeric enzymes in vivo and in vitro, providing new ideas and methods for immobilization of enzyme without carrier.
\end{abstract}

Keywords: Disassembly, iLID, Nitrilase, Photoswitch, Self-assembly

\section{Background}

Protein assembly has been extensively studied (King and Lai 2013; Yu et al. 2015), and represents a useful tool for biology and bioengineering processes owing to its potential applications in protein material development nanobiotechnology, and multienzyme biocatalysis (Matsumoto et al. 2016; Smith et al. 2011; Fairman and Åkerfeldt 2005; Holmes 2002). Although various protein assembly strategies have been reported, such as coimmobilization, protein fusion, protein crosslinking, and scaffold-mediated multienzyme colocalization (King and Lai 2013; Schoffelen and van Hest 2012; Brady and Jordaan 2009), those strategies do not have the ability to achieve the precise

\footnotetext{
*Correspondence: yhren@ecust.edu.cn

${ }^{4}$ State Key Laboratory of Bioreactor Engineering, New World Institute of Biotechnology, East China University of Science and Technology, Shanghai, China

Full list of author information is available at the end of the article
}

control of the assembly process, or realize the reversible disassembly in cell-free solutions (in vitro) or in bacterial cell cultures (in vivo). Including a control mechanism would solve this problem. Optogenetics provides a powerful tool for controlling biological processes through several light receptors such as cryptochromes (Kennedy et al. 2010), phytochrome B, phototropins (LOV), and rhodopsins (Levskaya et al. 2009; Yin and Wu 2013). Thus, we aimed to use light for the highly tunable, nontoxic, simple, and high-resolution spatiotemporal control of protein assembly. We focused on using the photosensitive LOV2 domain from Avena sativa (oat) (AsLOV2) (Strickland et al. 2010). AsLOV2 consists of a core PerArnt-Sim (PAS) domain that is sensitive to blue light and a $\mathrm{C}$-terminal helical extension $(\mathrm{J} \alpha)$. Upon irradiation with 470-nm blue light, a covalent adduct is generated between a conserved cysteine residue in the Per-ArntSim core of AsLOV2 and flavin mononucleotide (FMN) $\mathrm{C}(4 \mathrm{a})$, leading to the unfolding of the J $\alpha$ helix (Halavaty 
and Moffat 2007; Harper et al. 2004; Guntas et al. 2014). Several strategies have been developed to utilize AsLOV2 as a photoswitch, including using tunable light-inducible dimerization tags to control the interaction between the AsLOV2 domain and an engineered PDZ domain, and using the improved light-inducible dimer (iLID) system to control the location of transmembrane proteins (Guntas et al. 2014). The iLID system consists of an AsLOV2 mutant domain fused to the N-terminal of SsrA (called iLID-micro) and a $13-\mathrm{kDa}$ adaptor protein, $\mathrm{SspB}$, that can dimerize spontaneously and bind to SsrA (Guntas et al. 2014). After irradiation with 470-nm blue light, the conformation of the AsLOV2 domain changes, leading to the exposure of SsrA, which subsequently binds to SspB (Guntas et al. 2014; Zimmerman et al. 2016). The iLID presents several advantages, such as possessing strictly monomeric components, being easily expressed in Escherichia coli, and having a broad dynamic range, a highly tunable affinity, and a fast reversion rate in the dark (Guntas et al. 2014).

In this study, the dodecamer nitrilase from Burkholderia cenocepacia J2315 (Wang et al. 2013) (BCNIT) was selected as the model enzyme, and a light-controllable enzyme assembly method was developed using iLID and BCNIT. Dynamic light scattering (DLS), fluorescence complementation, scanning electron microscopy (SEM), optical density measurements $\left(\mathrm{OD}_{600}\right)$, and enzyme activity assays were performed to study the mechanisms involved.

\section{Methods}

\section{Materials}

PrimeSTAR $^{\circledR}$ Max DNA polymerase (Takara Biotech, Dalian, China) was used for PCR amplification. The sequence information for AsLOV2-SsrA and SspB was acquired from the Protein Data Bank (4WF0 and 1YFN) and the genes and primers were synthesized (Generay, Shanghai, China) with codon optimized for expression in E. coli. FMN, mandelonitrile, and mandelic acid were purchased from Sigma-Aldrich (Shanghai, China). All other chemicals were purchased from SinopHarm Chemical Reagent Co., Ltd. (Shanghai, China). The BCNIT gene was acquired from our laboratory.

\section{Construction of fusion genes}

The plasmids pET28a/BCNIT-AsLOV2-SsrA and pET28a/ SspB were constructed by PCR cloning using PrimeSTAR ${ }^{\circledR}$ Max DNA polymerase. The oligonucleotide sequence of the AsLOV2-SsrA domain containing $5^{\prime}$ SpeI and $3^{\prime}$ XhoI restriction sites was constructed on pET28a, and the flexibility linker of (GGGGS) $)_{2}$ was modified on the $\mathrm{N}$ terminal of AsLOV2-SsrA using the primers P3/R3. The BCNIT gene with $5^{\prime} \mathrm{NdeI}$ and $3^{\prime}$ SpeI restriction sites was amplified by PCR based on the BCNIT template using the primers $\mathrm{P} 2 / \mathrm{R} 2$. The genes were sequentially inserted into a modified pET28a plasmid with SpeI restriction sites, producing the pET28a/BCNIT-AsLOV2-SsrA (BNAS) domain, and then pET21a/BCNIT-AsLOV2-SsrA was constructed by inserting the BNAS domain into pET21a. The SspB gene was prepared by gene synthesis and constructed on pET21a and a pET21c plasmid modified with a resistance gene against chloromycetin instead of ampicillin. The sequences $\mathrm{mN} 159$ and $\mathrm{mC160}$ were inserted into pET21a/BCNIT-AsLOV2-SsrA and pET28a/BCNITAsLOV2-SsrA using the primers P4/R4 and P5/R5 to produce the plasmids pET21a/BCNIT-mN159-AsLOV2-SsrA (BNMnAS) and pET28a/BCNIT-mC160-AsLOV2-SsrA (BNMcAS), respectively. The plasmids were transfected into E. coli BL21 (DE3) for recombinant protein expression. All the primers used are shown in Table 1. The detail information of construction of fusion genes is described in the supporting information (See Additional file 1).

\section{Protein expression and purification}

Recombinant E. coli BL21 (DE3) cells were cultured in Luria-Bertani medium at $37{ }^{\circ} \mathrm{C}$ until reaching an optical density at $600 \mathrm{~nm}\left(\mathrm{OD}_{600}\right)$ of $0.5-0.7$. Protein expression was induced by adding $0.1 \mathrm{mM}$ isopropyl $\beta$-D-1-thiogalactopyranoside and incubating the cells at $18{ }^{\circ} \mathrm{C}$ for $20 \mathrm{~h}$. The BCNIT, BNAS, and SspB proteins were purified with $\mathrm{Ni}$-nitrilotriacetic acid columns (GE Healthcare, Waukesha, WI, USA). Protein concentrations were determined using the Bradford assay (Beyotime Biotechnology, Shanghai, China).

\section{Optical control of enzyme assembly and disassembly in $E$. coli}

The assembly of the fusion proteins in E. coli was monitored using the fluorescence complementation assay (Gao et al. 2015). The three plasmids pET21a/BNMnAS, pET28a/BNMcAS, and pET21c/SspB were cotransfected into E. coli BL21 (DE3). After centrifugation at $8000 \times g$

Table 1 Primers used for cloning and transcript amplification in this study

\begin{tabular}{ll}
\hline Primer & Sequence $\left(\mathbf{5}^{\prime} \mathbf{-} \mathbf{3}^{\prime}\right)$ \\
\hline P1 & GGAATTCCATATGGAATACAAATCCTC \\
R1 & CCGCTCGAGTTATTCATCGTAGATTTCTTCAG \\
P2 & GGAATTCCATATGACCATCAATCACCCG \\
R2 & GACTAGTCGAGCCACCGCCACCAGCGGGTGGACGCGC \\
P3 & GGACTAGTGGCGGTGGGGATCTTTAGCCACTACTTTAGAAAGG \\
R3 & ATAAGAATGCGGCCGCAAAATAATTTTCATCATTAG \\
P4 & AGGTGGCTCTACTAGTGGCGAGGTGGTCTGTG \\
R4 & CACCTCCGCCACTAGTCGATCCGCCACCGCCGTC \\
P5 & AGGTGGCTCTACTAGTGGCGGTGGCGATCTGGC \\
R5 & CACCTCCGCCACTAGTAGAGCCACCTCCGCCGCT
\end{tabular}


for $10 \mathrm{~min}$ and resuspension of the cell pellets in $5 \mathrm{~mL}$ $20 \mathrm{mM}$ phosphate-buffered saline (PBS; pH 7.4), $10 \mu \mathrm{M}$ FMN was added and the cells were incubated for $10 \mathrm{~min}$ at $37{ }^{\circ} \mathrm{C}$ under a $470-\mathrm{nm}$ blue light-emitting diode array (Guntas et al. 2014). Meanwhile, an equivalent number of cells coexpressing BNMnAS, BNMcAS, and SspB in the dark, and cells coexpressing $\mathrm{mN159}, \mathrm{mC160}$, and SspB under 470-nm light were used as controls and assayed in the same manner. We monitored the differences between the samples placed under the blue light and those kept in the dark by measuring epifluorescence (excitation: 562$640 \mathrm{~nm}$, emission: 590-650 nm).

\section{Optical control of enzyme assembly and disassembly in vitro}

The BNAS fusion proteins were assembled on SspB by triggering the affinity between AsLOV2-SsrA and SspB under illumination at $470 \mathrm{~nm}$. Self-assembly via photopolymerization occurred in PBS buffer ( $\mathrm{pH} 7.4)$ after mixing the freshly purified BNAS and SspB proteins at an equimolar ratio under freezing conditions and adding $10 \mu \mathrm{M}$ FMN to induce a stable supramolecular polymerization. The disassembly measurement was performed in the dark after 470-nm blue light illumination. The optical density at $600 \mathrm{~nm}\left(\mathrm{OD}_{600}\right)$ was used to characterize the turbidity of the supramolecular assembly (Kanekura et al. 2016). The reactions before measured the $\mathrm{OD}_{600}$ were carried out in an ice bath. Moreover, the assemblies were visualized using scanning electron microscopy. BNAS or BCNIT and SspB were mixed at a 1:1 molar ratio.

\section{Dynamic light scattering (DLS) assay}

To explore the assembly and disassembly before and after illumination, we used a DynaPro NanoStar ${ }^{\circledR}$ instrument (Wyatt Technology, Santa Barbara, CA, USA) to analyze the changes in the particle size of the BNAS-SspB (BNASS) supramolecular complex. Samples were filtered with a pore size of $0.22 \mu \mathrm{m}$ prior to analysis, and each measurement was repeated three times at $18{ }^{\circ} \mathrm{C}$.

\section{Field-emission scanning electron (FESEM)}

The sample was repeatedly washed with deionized water and resuspended, dried under air, and applied onto a slide. Images were collected on an S4800 scanning electron microscope (Hitachi, Tokyo, Japan) operated at $15 \mathrm{kV}$. In order to more clearly observe morphology and structure of BNASS supramolecular assemblies, we collected images with different resolutions.

\section{Enzymatic activity assay in vitro and in vivo}

Nitrilase activity was measured using reverse-phase high-performance liquid chromatography by monitoring the decrease of mandelonitrile (substrate) or the increase of mandelic acid (product) at $210 \mathrm{~nm}$. The standard assay mixtures contained $20 \mathrm{mM}$ mandelonitrile, $10 \mu \mathrm{M}$ FMN, $100 \mathrm{mM}$ PBS (pH 7.4), and $6 \mu \mathrm{M}$ pure enzyme (in vitro) or $10 \mathrm{mg} / \mathrm{mL}$ of $E$. coli cells expressing nitrilase (in vivo). After reaction at $30{ }^{\circ} \mathrm{C}$ with agitation at $200 \mathrm{rpm}$ for $20 \mathrm{~min}, 100 \mu \mathrm{L}$ of $1 \mathrm{M} \mathrm{HCl}$ was added to stop the reaction, and centrifugation was performed at $13,000 \times g$ for $10 \mathrm{~min}$, before removing $500 \mu \mathrm{L}$ of sample for reversephase high-performance liquid chromatography analysis using a Zorbax ${ }^{\circledR}$ SB-Aq column $(250 \times 4.6 \mathrm{~mm}, 5 \mu \mathrm{m}$; Agilent Technologies, USA) at a detection wavelength of $210 \mathrm{~nm}$ (Ni et al. 2013). The detail information of enzymatic activity assay is described in the supporting information (See Additional file 1).

\section{Results and discussion}

The strategy for the light-controlled self-assembly of dodecamer nitrilase in vitro and in vivo is outlined in Fig. 1. The fusion protein BNAS could be assembled under 470-nm light, and the resulting assemblies could be disassembled in the dark.
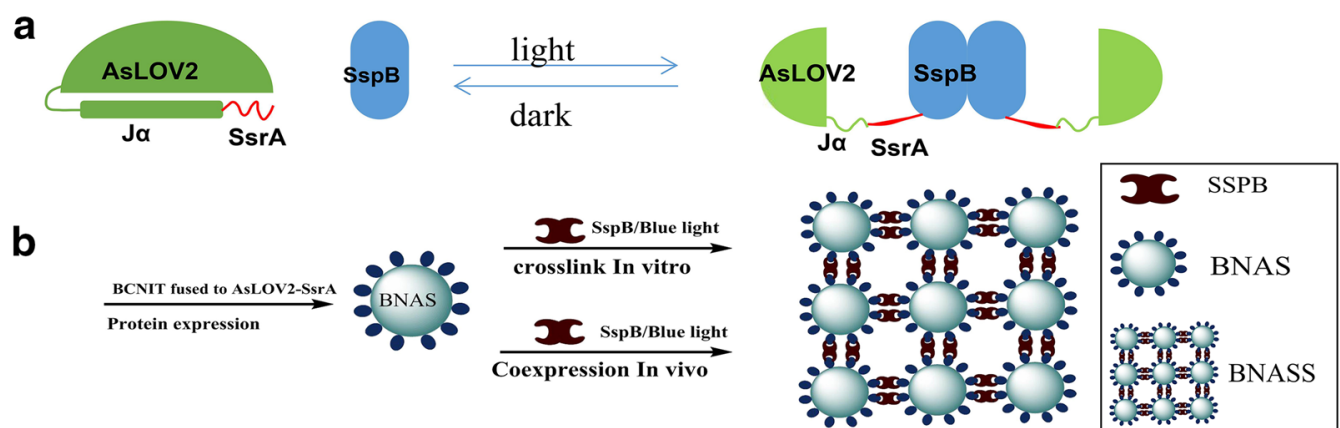

Fig. 1 Schematic design of assembly and disassembly. a Schematic design of the improved light-inducible dimer system. b Strategy for the light-controlled dodecamer assembly and disassembly of nitrilase from Burkholderia cenocepacia J2315 (BCNIT) in cell-free solution (in vitro) and in Escherichia coli (in vivo) 


\section{Light-controlled assembly and disassembly in vitro}

The in vitro light-controlled assembly was performed by expressing and purifying SspB and BNAS in E. coli BL21 (DE3). As shown in the Fig. 2a, the purified proteins were observed with the molecular mass of 13 and $58 \mathrm{kDa}$ for SspB and BNAS, respectively. BNAS retained about $97 \%$ of the specific activity of BCNIT (Fig. 2b), indicating that the protein fused with AsLOV2-SsrA did not suffer any drastic structural change in vitro. The light-controlled assembly was triggered by mixing the purified $\mathrm{SspB}$ and BNAS proteins with FMN under illumination at $470 \mathrm{~nm}$. Following illumination, the solution changed rapidly from clear to turbid (Fig. 3A), and the DLS measurements indicated that BNAS was converted into a BNASS supramolecular polymer (Fig. 3B). The hydrodynamic diameter of pure BNAS is about $30 \mathrm{~nm}$. The hydrodynamic diameter of the proteins decreased from 1000 to $100 \mathrm{~nm}$ after the light was removed, suggesting that the supramolecular polymer was disassembled within $5 \mathrm{~min}$. However, the assembly could not disassembled completely, which might result from a remnant weak affinity between SsrA and SspB in the dark.

The morphology and structure of BNASS supramolecular polymers were observed by scanning electron microscopy (SEM), and it was found that the assembly formed a circular sheet structure with a hydrodynamic diameter about $1 \mu \mathrm{m}$ (Fig. 3C). When the blue light was removed for $10 \mathrm{~min}$, the hydrodynamic diameter of the assembly was found to vary from 30 to $200 \mathrm{~nm}$ by SEM (Fig. 3D). The results confirmed that SspB and AsLOV2SsrA could mediate the formation of a supramolecular polymer under illumination at $470 \mathrm{~nm}$ and the polymer would be disassembled in the dark. Figure 4a shows the results obtained when monitoring the disassembly of the supramolecular polymer in the dark. The change of $\mathrm{OD}_{600}$ of the supramolecular polymer suggested that the supramolecular polymer disassembled dramatically, confirming the DLS results. An experiment was performed to examine whether different illumination intensities could influence the assembly under blue light and the disassembly in the dark. Figure $4 \mathrm{~b}$ shows that the $\mathrm{OD}_{600}$ increased gradually with the increasing illumination intensities. This result indicated that the light intensity has a great effect on the enzyme assembly, possibly because the light intensity affected the responding time of the AsLOV2-SsrA to the blue light, resulting in different degrees of assembly at the same time. However, when the light was removed, assemblies with different $\mathrm{OD}_{600}$ values showed varying degrees of disassembly (Fig. 4c). This observation could be explained by larger assemblies exhibiting a stronger clustering effect (Pieters 2009; Sengupta et al. 2011; Jaenicke 2000), thereby weakening the degree of disassembly. The disassembly efficiency is optimal when the $\mathrm{OD}_{600}$ is $0.7-$ 0.8 . Therefore, we employed the assembly with an $\mathrm{OD}_{600}$ value of 0.7 in the following experiments.

The recyclability of multienzyme polymers is an important factor when it comes to applications in synthetic biology and biocatalysis, especially for light-controlled enzyme assembly processes. The recyclability of the light-controlled assembly was evaluated by examining the change in the $\mathrm{OD}_{600}$ value of the mixture of BNAS and SspB when the mixture solution was repeatedly transferred between blue light illumination and the dark conditions. As shown in Fig. $4 d$, the $\mathrm{OD}_{600}$ value decreased from 0.7 to 0.1 within 5 min in the dark, then increased to 0.7 within a few seconds under illumination at $470 \mathrm{~nm}$, which is in accordance with the results displayed in Fig. 3B. Furthermore, neither the assembly nor

\section{a}

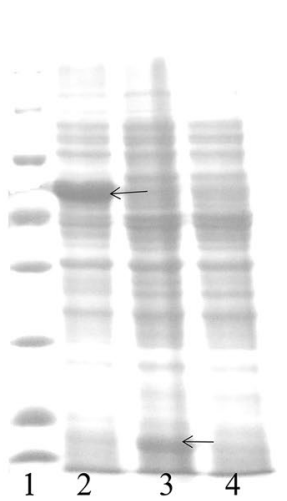

b

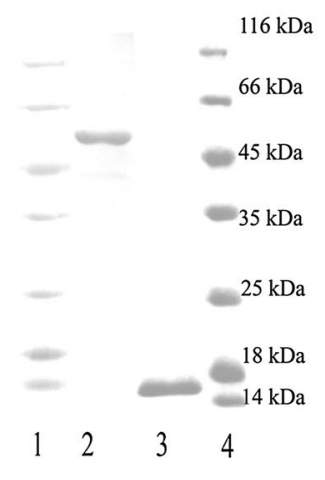

C

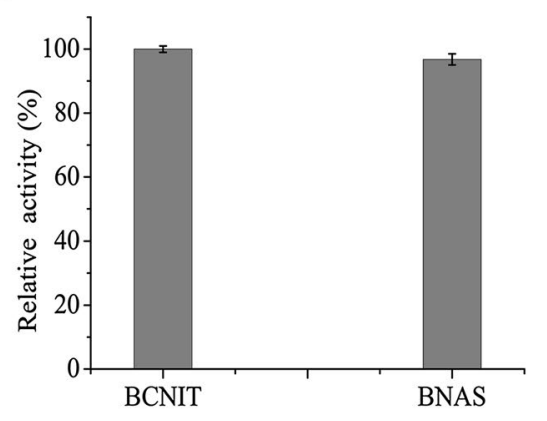

Fig. 2 Expressions of BNAS and SspB proteins in E. coli BL21 (DE3) cells. a SDS-PAGE image of the cells expressing BNAS and SspB at $20 \mathrm{~h}$ after IPTG addition. Lanes 1 marker; lane 2 BNAS; lane $3 \mathrm{SspB}$; lane 4 control. The arrow shows the destination band. b SDS-PAGE analyses of purified BNAS and $\mathrm{SspB}$ in vitro. Lanes 1 and 4 marker; lane 2 purified BNAS; lane 3 purified SspB. c Comparison of the enzyme activities of BCNIT and BNAS in vitro. The determination of nitrilase activity was performed at $30^{\circ} \mathrm{C}$ for $30 \mathrm{~min}$ with $10 \mu \mathrm{M}$ FMN under 470 -nm blue light illumination. Under the experimental conditions, the relative activity was expressed as a percentage of the maximum activity. Error bars represent the standard error of three replicates 

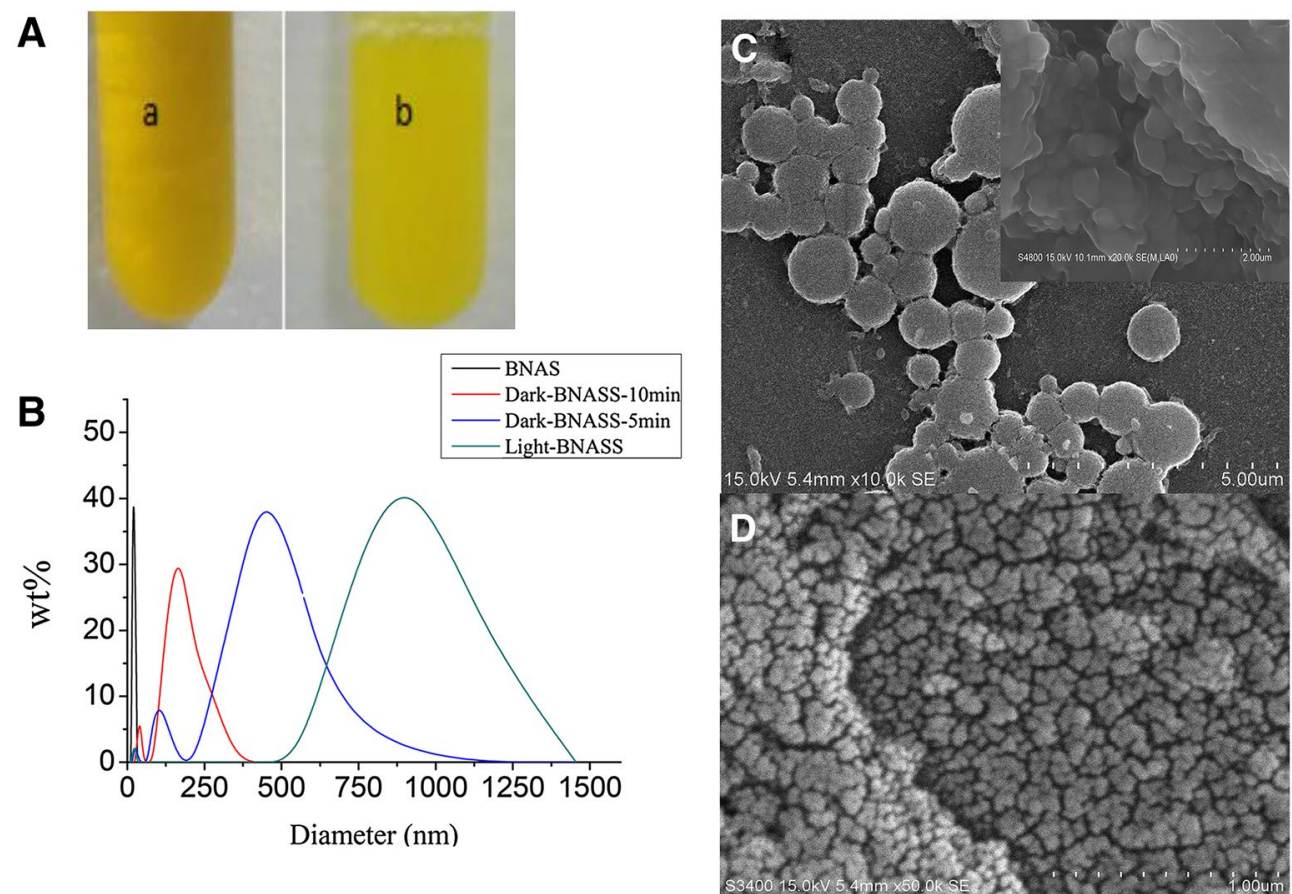

Fig. 3 The light-controlled assembly, disassembly, and morphology of the BCNIT-AsLOV2-SsrA-SspB (BNASS) complex in vitro. A The solution states of the mixture of BNAS, SspB, and FMN either (a) kept in the dark or (b) exposed to light. B Dynamic light scattering analysis of the hydrodynamic diameter of pure BCNIT-AsLOV2-SsrA (BNAS) and the mixture of SspB and BNAS. C Structure of the BNASS complex in vitro visualized by FESEM and SEM after illumination with blue light. D Structure of the BNASS complex in vitro visualized by SEM when the light is removed for 10 min. The reactions were carried out in an ice bath

the disassembly was affected by repeating the light/dark cycling up to five times.

To further determine whether the catalytic activity of the assemblies is affected by repeated assembly-disassembly cycles, we analyzed the activity of the supramolecular assembly through seven cycles of illumination at $470 \mathrm{~nm}$ followed by incubation in the dark. Figure 5 shows that the light-controlled assembly retained a high level of activity as the number of cycles increased, and still retained $90 \%$ of its initial activity after four cycles. The dramatic decrease of enzyme activity after five or more cycles may be caused by the disruption of the enzyme assembles after the violent resuspension, the loss of some smaller enzyme assembles during centrifugal isolation, and the toxicity of nitrile substrate after multicycles.

\section{Light-controlled assembly in vivo}

To assess whether the light-controlled assembly and disassembly of the fusion proteins can occur within $E$. coli cells, BNAS and SspB were coexpressed in E. coli BL21 (DE3), and both of them could be expressed in their soluble forms. To monitor the light-controlled formation of the assemblies in vivo, a fluorescence complementation assay was also performed. The red fluorescent protein $\mathrm{mCheery}$ was divided into two parts, $\mathrm{mN} 159$ and $\mathrm{mC160}$, and was fused to the site between BCNIT and AsLOV2-SsrA, thus producing the BCNITmN159-AsLOV2 (BNMnAS) and BCNIT-mC160AsLOV2 (BNMcAS), respectively. Little fluorescence was observed when mN159, mC160, and SspB were coexpressed in E. coli BL21 (DE3) and the cells were illuminated under blue light (Fig. 6a). However, the fluorescence intensity increased markedly when BNMnAS and BNMcAS were coexpressed with SspB under illumination at $470 \mathrm{~nm}$ (Fig. 6b), indicating that the strong fluorescence is due to the presence of AsLOV2-SsrA. Concordant with the increase of fluorescence intensity resulting from induction by blue light illumination, the strains coexpressing BNMnAS, BNMcAS, and $\mathrm{SspB}$ showed significantly lower fluorescence intensities in the dark (Fig. 6c). Although the core domain of the AsLOV2 covered the binding site of the interaction between SsrA and SspB in darkness, the SsrA domain was not completely inactivated, and hence SrA and SspB kept a weaker affinity in darkness (Guntas et al. 2014). The weaker affinity led to the assembly of fewer nitrilases, and thus there were weak fluorescent signals 

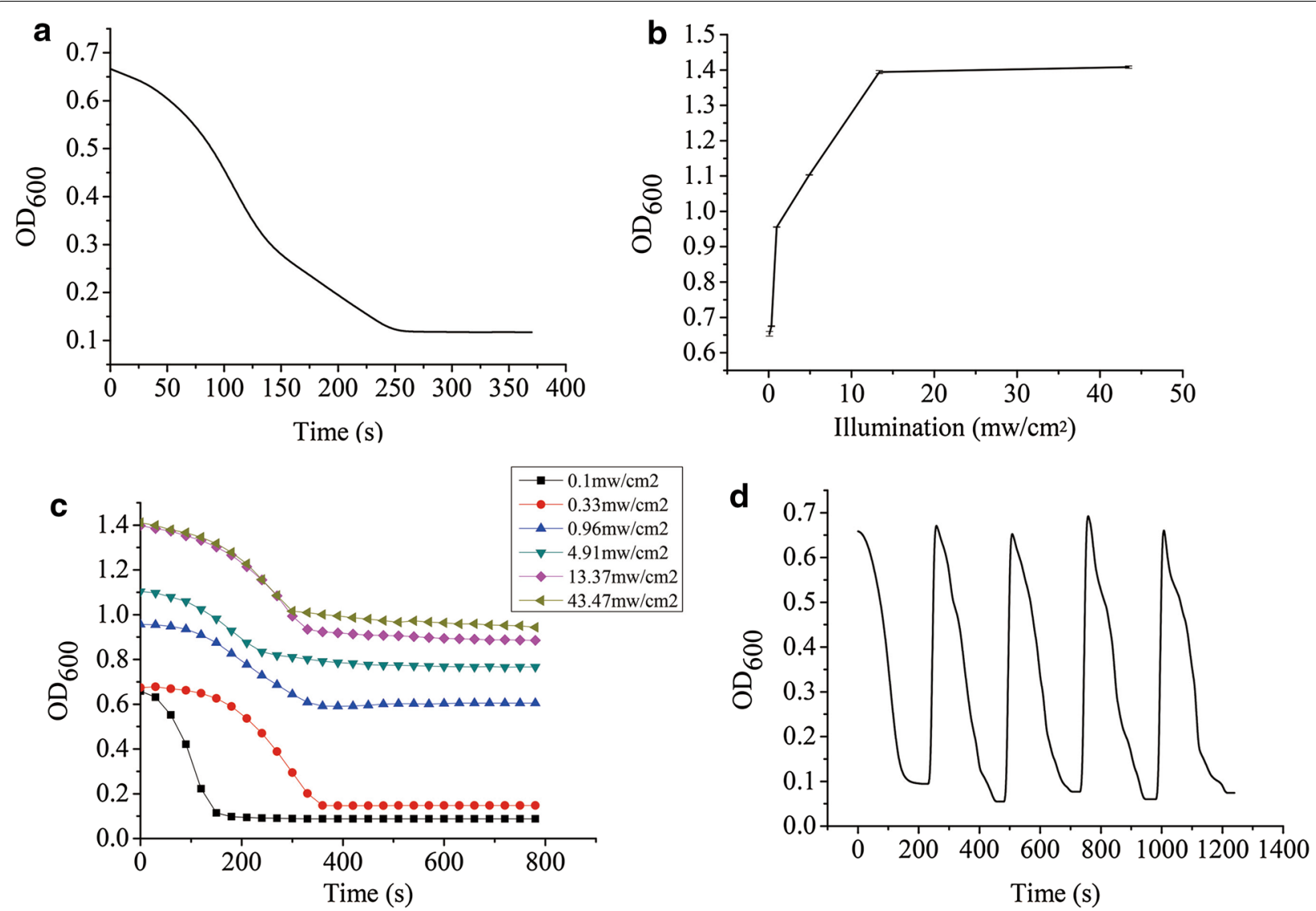

Fig. 4 Characterization of the light-controlled reversible assembly and disassembly of the BNAS in vitro by measuring the optical density at $600 \mathrm{~nm}$ and the nitrilase activity of BNASS. a Changes in $\mathrm{OD}_{600}$ values with the disassembly of the BNASS complex. $\mathbf{b}$ Changes in $\mathrm{OD}_{600}$ values under different blue light intensities during photopolymerization. $\mathbf{c}$ Different degrees of disassembly of the polymer incubated under dark conditions after illumination at different intensities. $\mathbf{d}$ Light-induced reversible assembly and disassembly of the BNASS complex. The reactions were carried out in an ice bath. Error bars represent the standard error of three replicates

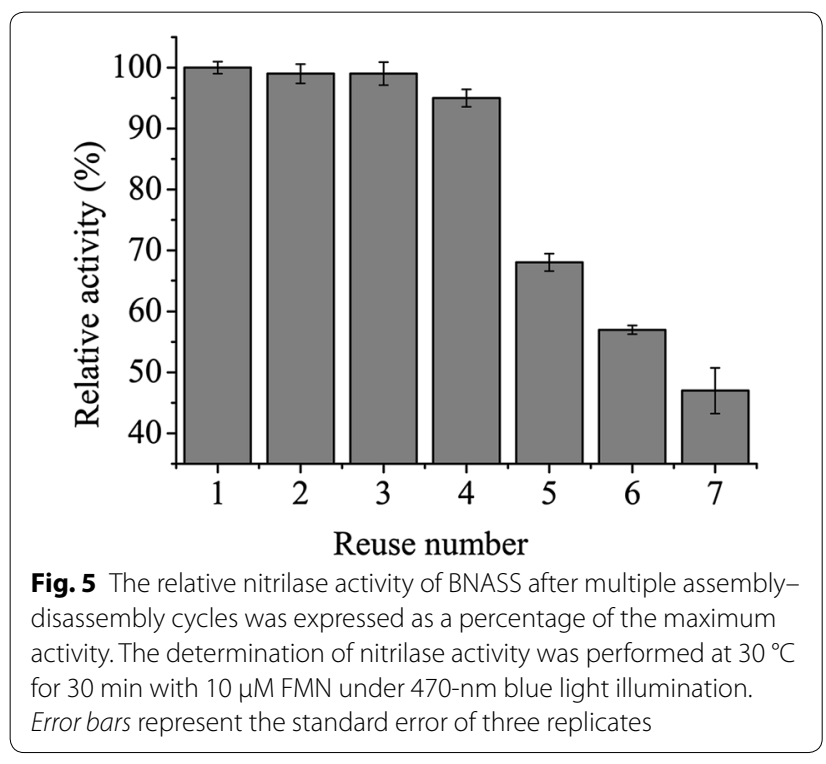

in the dark. At the same time, the fluorescence intensity of $5.0 \mathrm{OD}_{600}$ cells collected after induction was measured using a Spectra Max M5 microplate reader (Synergy Mx, Bio-Tek Instruments, Inc., Winooski, VT, USA). As shown in Fig. 7a, the results of fluorescence intensities are exactly the same as those shown in Fig. 6. All results showed that the strong fluorescence signal is due to the presence of AsLOV2-SsrA and blue light. To determine the catalytic activity of BNASS in vivo, BNAS and SspB were coexpressed in E. coli BL21. As a control, the same number of cells expressing BNAS and BCNIT were analyzed following the same procedure. Similar to the result obtained in vitro, as shown in Fig. $7 \mathrm{~b}$, the activity of BNAS in vivo remained at $93 \%$ relative to that of BCNIT. The supramolecular assembly BNASS also maintained approximately $85 \%$ activity relative to that of BCNIT regardless of light or dark exposure. The results show that the supramolecular assembly process barely changed the activity of BCNIT. 

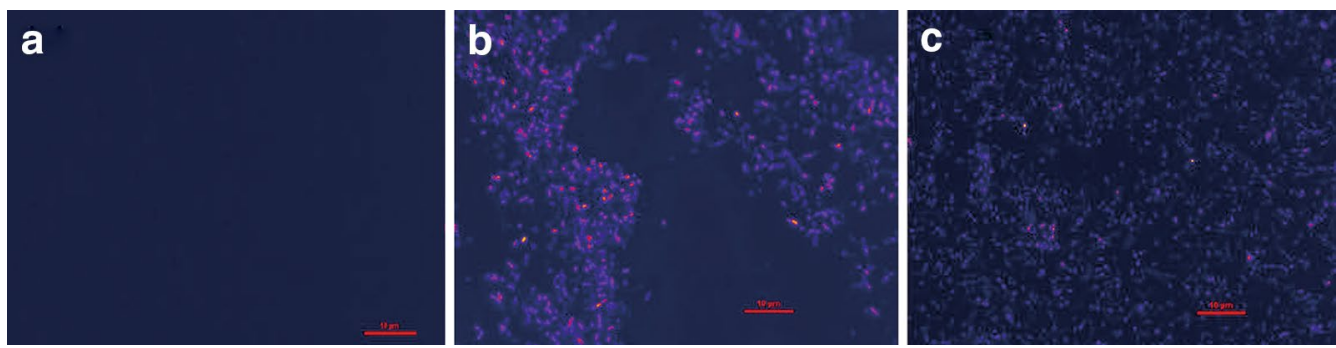

Fig. 6 Fluorescence microscopy visualization of Escherichia coli BL21 cells coexpressing a mN159, mC160, and SspB under blue light, b BNMnAS, BNMcAS, and SspB under blue light. c BNMnAS, BNMcAS, and SspB in the dark. Scale bar $10 \mu \mathrm{m}$
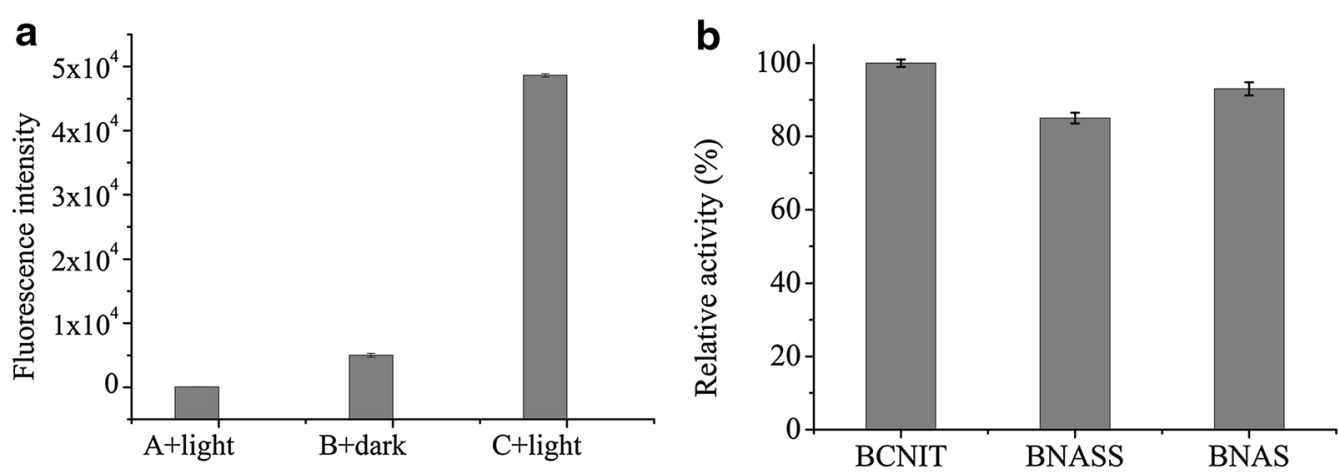

Fig. 7 The fluorescence intensities of fusion protein-coexpressing strains. a A + light represents the strains coexpressing mN159, mC160, and SspB under blue light illumination; B + dark represents the strains coexpressing BCNIT-mN159-AsLOV2-SsrA (BNMnAS), BCNIT-mC160-AsLOV2-SsrA (BNMCAS), and SspB in the dark; C + light represents the strains coexpressing BNMnAS, BNMcAS, and SspB under blue light illumination. b Comparison of the nitrilase activities of BCNIT, BNAS, and BNASS in E. coli. Error bars represent the standard error of three replicates. The determination of nitrilase activity was performed at $30^{\circ} \mathrm{C}$ for 30 min with $10 \mu \mathrm{M}$ FMN under 470-nm blue light illumination. Under the experimental conditions, the relative activity was expressed as a percentage of the maximum activity. Error bars represent the standard error of three replicates

\section{Conclusions}

In conclusion, a strategy for the light-controlled assembly and disassembly of oligomeric enzymes through an optogenetic tool was successfully applied to in vitro and in vivo systems. The light-controlled assembly did not affect the catalytic ability of enzymes, and the assemblies showed good reusability and reversibility. The results indicated that the construction of supramolecular assemblies mediated by AsLOV2 can provide a solid biocatalyst with good reusability and reversibility in cellfree solutions and bacterial cultures, and this method might provide a promising way to precisely and reversibly control protein-protein interactions in mammalian cells. These results indicate that the photocontrolled self-assembly strategy is a powerful tool for achieving the scaffold-free self-assembly and immobilization without carrier of enzymes. Thus, the photocontrolled enzyme assembly strategy may be used in the multienzyme biocatalysis to realize precise and reversible control of the process of metabolic enzyme cascades in vivo and in vitro.

\section{Additional file}

Additional file 1. Supporting Information.

\section{Abbreviations}

AsLOV2: LOV2 domain isolated from Avena sativa; BCNIT: nitrilase from Burkholderia cenocepacia J2315; BNAS: BCNIT-AsLOV2-SsrA domain; BNASS: BNAS-SspB; BNMnAS: BCNIT-mN159-AsLOV2-SsrA; BNMCAS: BCNIT-mC160AsLOV2-SsrA; CRY: cryptochromes; DLS: dynamic light scattering; FMN: flavin mononucleotide; HPLC: high-performance liquid chromatography; iLID: photoswitch-improved light-inducible dimer; IPTG: isopropyl $\beta$-D-1thiogalactopyranoside; LB: Luria broth; LOV: light-oxygen-voltage; OD: optical density; PAS: Per-Arnt-Sim; PhyB: phytochrome B; FESEM: field-scanning electron microscopy.

\section{Authors' contributions}

QY conducted the experiments and the manuscript writing. YR provided advice in the experiments design and data analysis. All authors read and approved the final manuscript.

\section{Author details}

${ }^{1}$ East China University of Science and Technology, Shanghai, China. ${ }^{2}$ Key Laboratory of Synthetic Biology, Institute of Plant Physiology and Ecology, Shanghai Institutes for Biological Sciences, Chinese Academy of Sciences, Shanghai, China. ${ }^{3}$ Fujian Key Laboratory of Eco-Industrial Green Technology, 
Wuyi University, Wuyishan, China. ${ }^{4}$ State Key Laboratory of Bioreactor Engineering, New World Institute of Biotechnology, East China University of Science and Technology, Shanghai, China.

\section{Acknowledgements}

This work was funded by the National Special Fund for the State Key Laboratory of Bioreactor Engineering (2060204).

\section{Competing interests}

The authors declare that they have no competing interests.

\section{Availability of data and materials}

The dataset supporting the conclusions of this article is available.

\section{Consent for publication}

The authors approved the consent for publishing the manuscript.

\section{Ethics approval and consent to participate}

All the authors have read and agreed the ethics for publishing the manuscript.

\section{Funding}

The State Key Laboratory of Bioreactor Engineering (2060204).

\section{Publisher's Note}

Springer Nature remains neutral with regard to jurisdictional claims in published maps and institutional affiliations.

Received: 12 May 2017 Revised: 23 July 2017 Accepted: 1 August 2017 Published online: 04 August 2017

\section{References}

Brady D, Jordaan J (2009) Advances in enzyme immobilisation. Biotechnol Lett 31(11):1639

Fairman R, Åkerfeldt KS (2005) Peptides as novel smart materials. Curr Opin Struct Biol 15(4):453-463

Gao X, Zhao CC, Yu T, Yang S, Ren YH, Wei DZ (2015) Construction of a reusable multi-enzyme supramolecular device via disulfide bond locking. Chem Commun 51(50):10131-10133

Guntas G, Hallett RA, Zimmerman SP, Williams T, Yumerefendi H, Bear JE, Kuhlman B (2014) Engineering an improved light-induced dimer (iLID) for controlling the localization and activity of signaling proteins. Proc Natl Acad Sci USA 112(1):112-117

Halavaty AS, Moffat K (2007) N- and C-terminal flanking regions modulate light-induced signal transduction in the LOV2 domain of the blue light sensor phototropin 1 from Avena sativa. Biochemistry 46(49):14001-14009

Harper SM, Christie JM, Gardner KH (2004) Disruption of the LOV-J alpha helix interaction activates phototropin kinase activity. Biochemistry 43(51):16184-16192

Holmes TC (2002) Novel peptide-based biomaterial scaffolds for tissue engineering. Trends Biotechnol 20(1):16-21
Jaenicke R (2000) Stability and stabilization of globular proteins in solution. J Biotechnol 79(3):193-203

Kanekura K, Yagi T, Cammack AJ, Mahadevan J, Kuroda M, Harms MB, Miller TM Urano F (2016) Poly-dipeptides encoded by the C9ORF72 repeats block global protein translation. Hum Mol Genet 25(9):1803-1813

Kennedy MJ, Hughes RM, Peteya LA, Schwartz JW, Ehlers MD, Tucker CL (2010) Rapid blue-light-mediated induction of protein interactions in living cells. Nat Methods 7(12):973-975

King NP, Lai YT (2013) Practical approaches to designing novel protein assemblies. Curr Opin Struct Biol 23(4):632-638

Levskaya A, Weiner OD, Lim WA, Voigt CA (2009) Spatiotemporal control of cell signalling using a light-switchable protein interaction. Nature 461(7266):997-1001

Matsumoto T, Isogawa Y, Minamihata K, Tanaka T, Kondo A (2016) Twigged streptavidin polymer as a scaffold for protein assembly. J Biotechnol 225:61-66

Ni KF, Wang HL, Zhao L, Zhang MJ, Zhang SY, Ren YH (2013) Efficient production of (R)-(-)-mandelic acid in biphasic system by immobilized recombinant E. coli. J Biotechnol 167(4):433-440

Pieters RJ (2009) Maximising multivalency effects in protein-carbohydrate interactions. Org Biomol Chem 7(10):2013-2025

Schoffelen S, van Hest JCM (2012) Multi-enzyme systems: bringing enzymes together in vitro. Soft Matter 8(6):1736-1746

Sengupta P, Jovanovic-Talisman T, Skoko D, Renz M, Veatch SL, LippincottSchwartz I (2011) Probing protein heterogeneity in the plasma membrane using PALM and pair correlation analysis. Nat Methods 8(11):969-975

Smith KH, Tejeda-Montes E, Poch M, Mata A (2011) Integrating top-down and self-assembly in the fabrication of peptide and protein-based biomedical materials. Chem Soc Rev 40(9):4563-4577

Strickland D, Yao X, Gawlak G, Rosen MK, Gardner KH, Sosnick TR (2010) Rationally improving LOV domain-based photoswitches. Nat Methods 7(8):623-626

Wang HL, Sun HH, Wei DZ (2013) Discovery and characterization of a highly efficient enantioselective mandelonitrile hydrolase from Burkholderia cenocepacia J2315 by phylogeny-based enzymatic substrate specificity prediction. BMC Biotechnol 13(1):14-24

Yin TF, Wu YI (2013) Guiding lights: recent developments in optogenetic control of biochemical signals. Pfluegers Arch 465(3):397-408

Yu T, Gao X, Ren YH, Wei DZ (2015) Assembly of cellulases with synthetic protein scaffolds in vitro. Bioresour Bioprocess 2(1):16-22

Zimmerman SP, Hallett RA, Bourke AM, Bear JE, Kennedy MJ, Kuhlman B (2016) Tuning the binding affinities and reversion kinetics of a light inducible dimer allows control of transmembrane protein localization. Biochemistry 55(37):5264-5271

\section{Submit your manuscript to a SpringerOpen ${ }^{\odot}$ journal and benefit from:}

- Convenient online submission

- Rigorous peer review

- Open access: articles freely available online

- High visibility within the field

- Retaining the copyright to your article 